\title{
The Role of Lipid Physical Properties in Lipid Barriers ${ }^{1}$
}

\author{
ALLEN G. Gibis ${ }^{2}$ \\ Evolutionary and Comparative Physiology Group, Department of Ecology and Evolutionary Biology, \\ University of California, Irvine, California 92697-2525
}

\begin{abstract}
SYNOPSIS. The hydrophobic nature of lipids means that they provide good barriers to the movement of charged and polar molecules. Barrier function appears to depend on the physical state of the lipids. Two well-investigated examples include cell membranes and epicuticular lipids of arthropods. Ecologically relevant changes in temperature significantly affect lipid properties, and both evolutionary and acclimatory differences in lipid composition appear to preserve the physical properties of lipids under different environmental conditions. These differences are generally believed to be beneficial to the organism, but rigorous examination of their adaptive significance is rare. Important issues are how lipid properties are regulated; which properties are physiologically relevant, how are these properties sensed, and what biochemical and molecular mechanisms regulate lipid properties? Progress has recently been made in understanding how membrane lipid properties are regulated, but regulatory mechanisms for cuticular lipids and other lipid systems remain completely unknown.
\end{abstract}

\section{INTRODUCTION}

Lipids comprise those compounds that are insoluble in aqueous media and soluble in non-polar solvents. They are unique among biological molecules in that they are defined by their physical properties rather than by their chemical structure. Unlike proteins and nucleic acids, which are simply polymers of a limited set of smaller molecules, lipids span a range of chemically distinct forms (Hadley, 1985; StanleySamuelson and Nelson, 1993). This diversity contributes to the wide range of biological functions in which lipids are involved, from major structural elements of cells to chemical signals within and among individuals.

In many cases, physiological functions of lipids appear to depend upon their physical properties. The most well-documented examples concern lipids that serve as barriers (e.g., cell membranes, cuticular lipids of arthropods). Ecologically relevant changes in environmental conditions such as temperature have substantial effects on the physical

\footnotetext{
1 From the Symposium The Biology of Lipids: Integration of Structure and Function presented at the Annual Meeting of the Society for Integrative and Comparative Biology, 26-30 December 1996, Albuquerque, New Mexico.

2E-mail: agibbs@uci.edu
}

properties of lipids. Membranes and cuticular lipids share the fact that their composition and properties appear to be closely regulated. The primary focus of this review is on the maintenance of lipid-based physiological processes in the face of environmental stress, although I emphasize at the outset that much of the evidence for the importance of lipid properties is only correlative. This situation is beginning to change, as new techniques from molecular and evolutionary biology are applied to these systems.

\section{Physical Properties of Cellular Membranes}

Cell membranes are by far the most thoroughly studied lipid systems. In the presence of water, phospholipids spontaneously assemble into ordered bilayer membranes that are held together by hydrophobic interactions between acyl chains (Cevc, 1991). The insolubility of lipids in polar solvents carries the converse implication that polar and charged compounds will be insoluble in lipids. Thus, membrane bilayers form excellent barriers to the movement of water and ions into and out of the cell. The flow of molecules and information across the cell membrane is regulated by membrane proteins. An important issue in 
membrane biology is the extent to which changes in the membrane lipid environment affect the function of transport and receptor proteins (Cossins, 1994; Gibbs, 1995). In a few cases, proteins require specific lipids in order to function (e.g., Robinson and $\mathrm{Ca}$ paldi, 1977). Such specificity is rare, and most proteins will function to some extent in a wide range of membrane environments. However, it is clear that not all membranes are equal, and that membrane proteins are affected by the types of lipids around them.

\section{Membrane fluidity and membrane protein function}

Physiologically relevant decreases in temperature or increases in pressure make cell membranes less fluid (more viscous; Hazel and Williams, 1990; Cossins, 1994). Studies using purified $\mathrm{Na} / \mathrm{K}$-adenosine triphosphatase ( $\mathrm{Na} / \mathrm{K}$-ATPase) in defined lipid environments have shown that enzyme activity is highly correlated with membrane fluidity (Chong et al., 1985; Harris, 1985). Similar results have been obtained using other proteins (Squier et al., 1988). Thus, the effects of temperature on fluidity may affect ion transport and other cellular processes mediated by membrane proteins.

The direct effects of temperature on membrane fluidity can be offset by changing the composition of the membrane lipids. In most membrane systems studied to date, acclimation to lower temperatures results in increased fluidity, due primarily to increased unsaturation of fatty-acyl chains of phospholipids (Hazel and Williams, 1990). This response has been termed homeoviscous adaptation, to emphasize the apparent importance of maintaining constant membrane microviscosity (Sinensky, 1974). The term "adaptation" is used here in a broad sense, including both genetic adaptation among species and phenotypic responses to the environment within an individual's lifespan (Bennett, 1997). The important question, of course, is whether homeoviscous responses are in fact adaptive (i.e., beneficial to the organism).

Homeoviscous adaptation is the most widespread cellular response to temperature known, and has been demonstrated in all taxa studied to date. Even the polycyclic

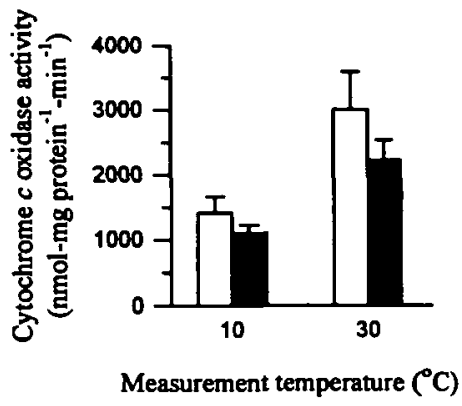

FIG. 1. Effects of temperature and thermal acclimation on the activity of cytochrome $c$ oxidase from carp. Cytochrome oxidase activity of mitochondrial membranes was assayed at 10 and $30^{\circ} \mathrm{C}$. Fish were acclimated to either $10^{\circ} \mathrm{C}$ (open bars) or $30^{\circ} \mathrm{C}$ (filled bars). Data from Table 1 in Wodtke (1981).

membrane-spanning ether lipids of thermophilic archaebacteria exhibit acclimatory responses consistent with homeoviscous adaptation (Kaneshiro and Clark, 1995). According to homeoviscous theory, increases in membrane fluidity at low temperature should result in increased enzyme catalytic rates and help to compensate for the inhibitory effects of reduced temperature. However, few studies have tested this hypothesis directly, because of difficulties in distinguishing between changes in the number of enzyme molecules and the per-molecule turnover rate. In one such study, acclimation to low temperature resulted in increased unsaturation and fluidity of mitochondrial inner membranes from carp (Wodtke, 1981). These changes were correlated with increased specific activity of an inner membrane enzyme, cytochrome $c$ oxidase, such that enzyme turnover rates were $30-35 \%$ higher in individuals acclimated to low temperature (Fig. 1). These results support the idea that homeoviscous adaptation affects cytochrome oxidase activity, but other factors (e.g., post-translational modifications of the protein) could also have been involved (Poly, 1997).

Manipulation of the membrane environment provides a more direct test of the influence of membrane fluidity on enzyme properties. Trout acclimated to low temperature exhibited increase turnover rates of erythrocyte $\mathrm{Na} / \mathrm{K}$-ATPase and increased membrane fluidity (Raynard and Cossins, 


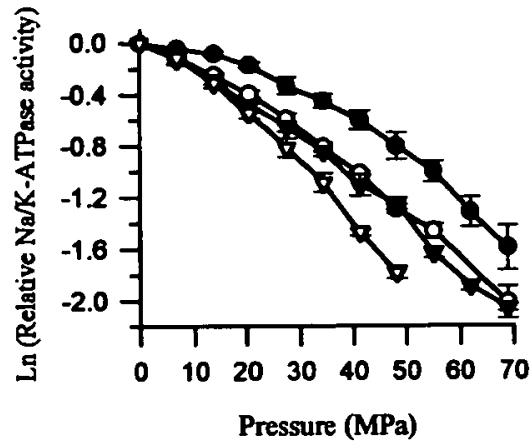

FIG. 2. Effects of pressure on gill $\mathrm{Na} / \mathrm{K}$-ATPase activity in marine fishes. Pressure increases by approximately $1 \mathrm{MPa}$ for each $100 \mathrm{~m}$ increase in depth. The average depth of the ocean is $-3800 \mathrm{~m}$ (Gibbs, 1997), so pressures $>10 \mathrm{MPa}$ are typical of deep-sea environments. Species (common name, habitat depths, temperature range) were: ()$^{\circ}$ Coryphaenoides armatus (rattail, $1,900-4,800 \mathrm{~m}, 2-4^{\circ} \mathrm{C}$ ), (O) C. acrolepis (rattail, $700-1,820 \mathrm{~m}, 4-8^{\circ} \mathrm{C}$ ), ( $)$ Porichthys notatus (midshipman, 0-300 $\mathrm{m}, 6-15^{\circ} \mathrm{C}$ ) and $(\nabla)$ Sphyraena helleri (barracuda, $0-20 \mathrm{~m}, 24^{\circ} \mathrm{C}$ ). Data from Gibbs and Somero (1989).

1991). Cholesterol supplementation reduced both fluidity and $\mathrm{Na} / \mathrm{K}$-ATPase activity to an extent similar to thermal acclimation, suggesting that acclimation of membrane fluidity helps to maintain ion transport rates under varying temperatures.

A potential problem with interpreting thermal effects on membrane proteins is that temperature has large effects on nearly all biochemical processes. Thus, it can be difficult to distinguish the direct effects of temperature on membrane proteins from indirect effects mediated by membrane lipids. Hydrostatic pressure provides one means of altering membrane fluidity without changing temperature. The direct effects of hydrostatic pressure on membrane properties are very similar to those of low temperature: reduced fluidity, increased thickness etc. Deep-sea organisms tend to have higher levels of unsaturated lipids that offset the membrane-ordering effects of high pressures (Gibbs, 1997). Because phospholipid bilayers are more sensitive to pressure (i.e., more compressible) than proteins, the effects of pressure on lipid-protein systems should be due largely to effects on the lipid component.

Pressure strongly inhibits $\mathrm{Na} / \mathrm{K}$-ATPase

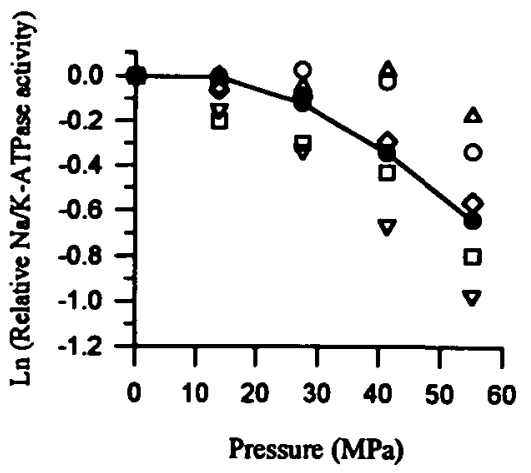

FIG. 3. Effects of lipid substitution on Na/K-ATPase from the deep-sea fish, Coryphaenoides armatus. The substitution procedure of Warren et al. (1974) was used to modify the lipid environment of $\mathrm{Na} / \mathrm{K}$-ATPase. Filled circles indicate the effects of pressure on ATPase activity in the enzyme's native lipid environment. Native lipids were replaced with: $(O)$ phospholipids (PLs) isolated from $C$. armatus; $(\triangle)$ PLs from a cold, shallow-living fish, Anoplopoma fimbria; $(\diamond)$ PLs from a warm-living fish, Sphyraena barracuda; $(\nabla)$ chicken egg phosphatidylcholine; and $(\square)$ a 1:1 mixture of cholesterol and A. fimbria PLs. Data from Gibbs and Somero (1990).

activity (Gibbs and Somero, 1989). Differences among species in pressure responses are correlated with presumed differences in membrane fluidity; sodium pump activity is least affected by pressure in deep-sea species, and most inhibited in species from warm, shallow habitats (Fig. 2). These effects can be altered by changing the membrane environment around the protein, using a lipid substitution procedure (Gibbs and Somero, 1990). Sodium pump activity is most inhibited by high pressure when the enzyme is placed in a relatively viscous, more saturated membrane environment, and least inhibited by pressure in the presence of relatively fluid lipids isolated from deepsea and cold-water fishes (Fig. 3). These results demonstrate that the catalytic properties of membrane enzymes can be directly affected by the membrane lipid environment, and therefore support the idea that homeoviscous adaptation is physiologically important.

Although fluidity has received the most attention from physiologists, many other physical properties of membranes are also affected by the environment. These include gel $\leftrightarrow$ fluid and bilayer $\leftrightarrow$ nonbilayer phase 
transitions, membrane thickness, and molecular cross-sectional area of phospholipids. Phospholipids in a fluid membrane have a greater molecular surface area due to increased molecular mobility, and the membrane as a whole will be thinner and will tend to freeze at lower temperatures. These correlations make distinguishing which properties of membranes are important a difficult experimental problem. Additionally, membrane properties may differ substantially within a given membrane, with consequent effects on membrane proteins (Post and Klodos, 1996; Williams, 1997). At even finer scales, membrane properties differ according to the depth in the bilayer, with sections closer to the middle of the bilayer being more fluid and having reduced lateral pressures (Scarlata, 1990; Cantor, 1997). Several recent authors have challenged the significance of membrane viscosity (e.g., Lee, 1991; Zakim et al., 1992; Hazel, 1995), and this remains an active area of research.

\section{Evolutionary and acclimatory changes in physical properties of membranes}

Organisms adapting over evolutionary time scales face differences in temperature similar to those experienced by eurythermal organisms, which need to acclimate to temperature within an individual's life span. Broadly speaking, differences in membrane composition between species from warm and cold habitats are similar to those of thermally-acclimated members of a given species. Animals from colder environments have relatively greater levels of lipid unsaturation in both cases, and their membranes are more fluid (Hazel and Williams, 1990).

An interesting contrast between evolutionary and acclimatory changes in membrane fluidity involves the relative "success" of homeoviscous adaptation in maintaining the same membrane fluidity at different temperatures. Comparison of vertebrate species from a wide range of body temperatures $\left(T_{b}\right)$ indicates that the fluidity of synaptosomal membranes is the same when measured at each species' $T_{b}$ (Behan-Martin et al., 1993). Thus, the "efficacy" of homeoviscous adaptation is 1 , i.e., evolutionary compensation for the ef- fects of temperature on membrane fluidity has been complete. Acclimatory responses are generally incomplete (i.e., the efficacy of homeoviscous adaptation is less than 1). However, these responses are highly variable, even among membrane fractions from the same cell. Some membranes exhibit no acclimation of fluidity at all (Lee and Cossins, 1990; Crockett and Hazel, 1995).

Biochemical mechanisms of homeoviscous adaptation within species have been examined for numerous organisms (Hazel and Williams, 1990). Changes in composition of phospholipid headgroups are involved in rapid acclimatory responses to temperature (Williams and Hazel, 1994). Changes in lipid saturation are slower, but occur rapidly enough to respond to diurnal variations in temperature (Carey and Hazel, 1989). In ectothermic vertebrates, a key enzyme in membrane acclimation is $\Delta^{9}$-desaturase, which catalyzes the insertion of a double bond in the 9-10 position of fattyacyl chains. Tiku et al. (1996), using carp, showed that changes in saturation during cold acclimation were associated with both increased synthesis of $\Delta^{9}$-desaturase and activation of existing desaturase molecules. In other taxa, activities of additional desaturases have been shown to increase during exposure to cold (Macartney et al., 1994).

An unexplored issue is whether similar biochemical mechanisms are involved in evolutionary differences among species and in acclimatory changes. Differences in membrane saturation are clearly implicated in both genetic and phenotypic variation, but is increased unsaturation in fishes living at cold temperatures due to higher basal levels of $\Delta^{9}$-desaturase, or are there additional biochemical differences? No consistent difference in headgroup composition has been demonstrated in inter-specific comparisons, which suggests evolutionary differences in membrane fluidity are not due to headgroup variation.

\section{Regulation of membrane properties}

Despite extensive investigation of the process and the results of homeoviscous adaptation, regulation of membrane properties is very poorly understood. Homeoviscous adaptation can be considered in terms of the 
feedback loops involved in many physiological processes. A regulated variable (which may or may not be membrane fluidity) must be sensed by some mechanism. The signal must be integrated and interpreted somehow, and the organism must respond by changing the amounts or activities of enzymes involved in membrane lipid synthesis. Detailed knowledge of any of these regulatory steps is lacking.

One way to identify the regulated variable in a feedback system is to manipulate suspected variables directly. Vigh et al. (1993) used a catalytic hydrogenation procedure to increase the saturation of membrane phospholipids in a strain of the cyanobacterium Synechocystis, which reduced membrane fluidity without changing temperature. Thus temperature-related changes in membrane properties and other cellular processes could be decoupled from changes in fluidity. Cells responded to reduced fluidity by increasing the expression of the $\operatorname{des} A$ gene product, a $\Delta^{12}$-desaturase. However, other membrane properties could also have been affected by the hydrogenation treatment. This study demonstrates that lipid saturation affects membrane biosynthesis, but does not distinguish among models for regulation of membrane properties.

How are membrane properties sensed? As noted above, it is unclear which membrane properties are essential for membrane function. Because various properties of membranes are correlated, the membrane property sensed by the cell may not be the membrane property which is physiologically most important. Integration of information regarding cell membrane properties is also almost completely unknown. Two lines of evidence indicate that integration is a sub-cellular phenomenom. First, isolated cells and cell cultures exhibit responses to temperature which are similar to those of tissues in intact organisms (Dey et al., 1993; Williams and Hazel, 1994). Second, different membrane systems within a cell may exhibit different acclimatory responses (Lee and Cossins, 1990; Crockett and Hazel, 1995), arguing against a general external signal regulating membrane synthesis.

Early work with the protozoan Tetrahymena pyriformis suggested that lipid desat- urases might be activated directly by membrane fluidity, so that membranes would be essentially self-regulating (Thompson and Nozawa, 1977). More recently, increased biosynthesis of desaturases at low temperatures has been found in several organisms (Macartney et al., 1994). In fishes, posttranslational modifications may also affect desaturase activity (Tiku et al., 1996). Molecular analyses of homeoviscous adaptation are still in the early stages, but results to date suggest that multiple regulatory mechanisms are operating.

\section{Is homeoviscous adaptation adaptive?}

The discussion above provides a minimal introduction into the large and diverse literature on homeoviscous adaptation. Ecologically relevant differences in temperature and other environmental variables significantly affect the physical properties of cell membranes, and these changes affect functional properties of membrane proteins. Acclimatory responses to changes in environmental temperature occur rapidly and involve differences in gene expression and possibly post-translational mechanisms, suggesting that membrane composition is closely regulated. The basic biochemistry of phenotypic acclimation and genetic adaptation to temperature is similar in a variety of organisms, suggesting a universal need to regulate membrane properties.

However, the organismal consequences of homeoviscous adaptation are very poorly understood. One could well ask whether homeoviscous adaptation really does benefit the organism. Would an organism's fitness or performance be compromised if homeoviscous adaptation did not occur? Because this appears to be a universal response to temperature, one can not investigate species which lack homeoviscous responses and compare them to those which do acclimate. A well-chosen taxon, including stenothermal and eurythermal species with a wide range of body temperatures, may provide an excellent system for the study of membrane adaptation and acclimation. All inter-specific studies to date have been performed without consideration of the phylogenetic relationships of the species involved. For example, Behan-Martin 


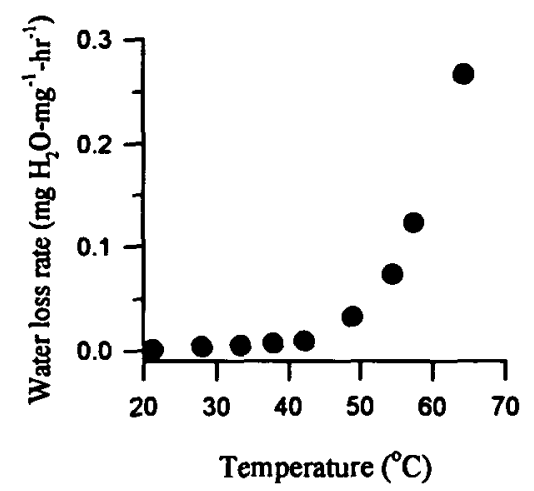

Fig. 4. Effects of temperature on rates of water loss from an individual grasshopper (Melanoplus sanguinipes). Water loss was measured by continuous weighing in a temperature-controlled microbalance. Cuticular lipids of $M$. sanguinipes melt at $38-50^{\circ} \mathrm{C}$ (Gibbs et al., 1991; Gibbs and Mousseau, 1994).

et al. (1993) included species ranging from passerine birds to Antarctic fishes.

A second approach to testing the physiological significance of homeoviscous adaptation would be to manipulate membrane composition and fluidity. This can be accomplished by direct chemical modification (Vigh et al., 1993) or by genetic techniques. For example, a mutant deficient in synthesis of desaturases should exhibit reduced thermal tolerance or other deficiencies at extreme temperatures. Animal models such as zebrafish and fruit flies have received very little attention from membrane physiologists, but could become useful study systems.

\section{Cuticular WATERPROOFING IN TERRESTRIAL ARTHROPODS}

Epicuticular lipids of terrestrial arthropods provide a second example for which the physical properties of lipids are thought to play an important physiological role. All terrestrial organisms face the problem of water loss through exposed surfaces, and all studied so far have evolved lipid layers which serve as the major barrier to evaporative water loss (Hadley, 1985, 1994). Insects and other small organisms are particularly vulnerable to dehydration, due to their relatively high surface area:volume ratio. Cuticular lipids in terrestrial arthropods have therefore been studied extensively

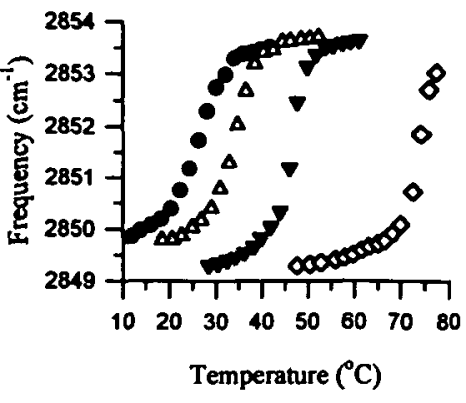

FIG. 5. Melting curves for cuticular lipids from four insect species. The frequency of $-\mathrm{CH}_{2}-$ symmetric stretching vibrations increases as lipids undergo the solid-fluid phase transition. From left to right, species were: Drosophila melanogaster, Blatella germanica. Melanoplus sanguinipes and Rhodnius prolixus.

(Blomquist et al., 1987; de Renobales et al., 1991). Most arthropods contain primarily long-chain hydrocarbons, but wax esters and other oxygenated compounds may predominate in some species (Buckner, 1993).

The ability of cuticular lipids to prevent water loss is thought to depend on their phase state. Rates of water loss from insects increase rapidly above a species-specific "critical" temperature (Fig. 4; Wigglesworth, 1945; Loveridge, 1968; Hadley, 1994). Early investigators suggested that increased transpiration is caused by the melting of the cuticular lipids. A number of biophysical studies, using various techniques, have found evidence consistent with this hypothesis (Beament, 1945; Holdgate and Seal, 1956; Toolson et al., 1979). A limitation of many experiments has been that surface lipids occur in very small quantities and have broad phase transitions. Thus, some biophysical techniques used in other systems may be inappropriate for these lipids. In some cases, only one species was investigated, so correlations between lipid melting and cuticular permeability may have been mere coincidence.

Recent work using infrared spectroscopy revealed extensive variation in lipid properties. Surface lipids melt at temperatures as low as $25^{\circ} \mathrm{C}$, although mealworm ( $\mathrm{Te}$ nebrio molitor) lipids remain solid up to $80^{\circ} \mathrm{C}$ (Fig. 5; Gibbs and Crowe, 1991). Thus, lipid melting occurs at physiologically relevant temperatures in some, but not all, species. Variation in melting point $\left(T_{m}\right)$ 


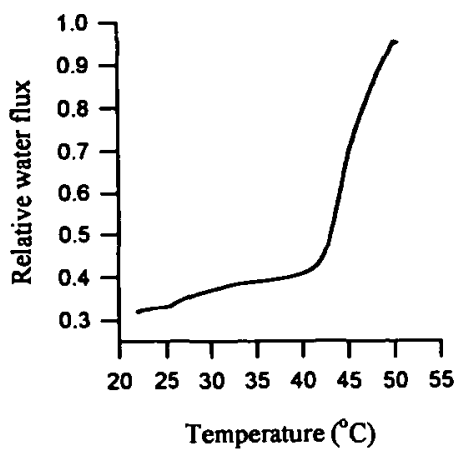

FIG. 6. Effects of temperature on water flux through a model membrane. A Gore-Tex membrane was coated with $n$-tricosane $\left(T_{\mathrm{m}}=42-44^{\circ} \mathrm{C}\right)$, and water flux through the membrane was measured using a flowthrough system.

exists within species as well. Grasshoppers (Melanoplus sanguinipes) exhibit geographic variation correlated with latitude, and $T_{m}$ is higher in individuals acclimated to warmer temperatures (Gibbs et al., 1991; Gibbs and Mousseau, 1994). This variation can be used to examine the questions of whether the properties of surface lipids really do affect rates of water loss, and which differences in lipid composition are likely to affect water balance.

I emphasize that the relationship between lipid melting and cuticular permeability still needs to be clearly resolved. In general, the evidence is favorable; organismal measurements, made using a variety of techniques (Figure 4; Beament, 1945; Toolson et al., 1979), and model cuticles (Fig. 6) exhibit similar transitions in water flux at the melting temperature of the lipid barrier. Another important issue is whether arthropods encounter environmental temperatures high enough to affect lipid physical properties. In $M$. sanguinipes, field body temperatures can exceed $40^{\circ} \mathrm{C}$, which would be high enough to melt cuticular lipids partially (Chappell, 1983; Gibbs et al., 1991). Together, these results suggest that increased rates of water loss associated with lipid melting can occur under ecologically relevant conditions.

\section{Genetic and acclimatory variation in} cuticular lipid properties

The cuticular lipids of arthropods exhibit such great diversity that broad comparisons across disparate taxa are largely uninformative. Only a few sets of closely related species have been used in comparative studies of the relationship between lipid composition and rates of water loss. Species with longer-chain hydrocarbons generally lose water less rapidly (Hadley, 1978; Hadley and Schultz, 1987). Unfortunately, the phylogenetically-based approaches developed in recent years have not yet been applied to insect water balance, but future work will certainly need to take evolutionary relationships into consideration.

Recent studies have demonstrated geographic variation in surface lipid composition (Ferveur et al., 1996). These differences have been shown to have a genetic basis, but the effects of this variation on water balance have not been investigated. In $M$. sanguinipes, populations from southern California tend to have lipids which melt at higher temperatures than those from northern populations (Gibbs et al., 1991). Recent evidence indicates that individuals from southern populations lose water less rapidly at the same measurement temperature (Rourke, personal communication). This pattern is consistent with local adaptation to higher environmental temperatures and lower humidities at lower latitudes, although other, non-adaptive explanations can not be excluded (Endler, 1986).

Thermal acclimation of cuticular hydrocarbons has also been correlated with rates of water loss (Toolson and Hadley, 1979; Toolson, 1982). Despite the lack of biophysical information in these studies, they are also consistent with the idea that acclimation of lipid properties affects water balance. Lipids from warm-acclimated individuals tend to have longer chain lengths. These differences should result in higher melting temperatures of the surface lipids and reduced rates of water loss. This may not be the case; acclimation to high temperature in the desert fruit fly, D. mojavensis, results in longer-chain length hydrocarbons (Markow and Toolson, 1990), but these changes in lipid composition are not associated with changes in $T_{\mathrm{m}}$ or rates of water loss (Gibbs et al., 1998). In M. sanguinipes, acclimation to warm temperatures actually results in relatively greater synthe- 
sis of shorter-length, unbranched hydrocarbons (Gibbs and Mousseau, 1994). Because straight-chain lipids melt at higher temperatures than branched lipids (Gibbs and Pomonis, 1995), overall $T_{\mathrm{m}}$ values are greater in warm-acclimated individuals (Gibbs et al., 1991; Gibbs and Mousseau, 1994).

Total lipid quantities also usually increase at higher temperatures, so it is often unclear whether higher- $T_{\mathrm{m}}$ lipids provide a better water-proofing barrier or whether the thickness of the barrier is the main factor determining cuticular permeability. Similar concerns apply in inter-specific comparisons. Both lipid amounts and lipid properties probably affect cuticular permeability.

\section{Regulation of Cuticular Lipid Properties}

The mechanisms whereby insects sense and maintain the properties of the cuticular lipids are completely unknown. It would seem beneficial for an insect to be able to detect and assess the physical properties of its surface lipids, but no such sensory mechanism has been described. In the case of mealworms and some other species, lipids melt at such high temperatures $\left(>80^{\circ} \mathrm{C}\right.$; Gibbs and Crowe, 1991) that no regulation would seem necessary. The surface lipids remain in a completely solid state at all non-lethal temperatures. In other species, such as $M$. sanguinipes and some Drosophila, melting temperatures may be in the environmental range (Chappell, 1983; Gibbs et al., 1997). One may then pose the question: Why don't all arthropods produce high- $T_{\mathrm{m}}$ lipids? Low- $T_{\mathrm{m}}$ lipids would not be a problem for insects with ready access to water, but $M$. sanguinipes in California can be found in very hot, dry habitats, where free water is not available.

A potential benefit of low- $T_{\mathrm{m}}$ surface lipids involves their dispersal over the cuticle. Cuticular lipids are deposited on the surface through pores, and in some species form visible wax blooms (Hadley, 1994). In others, the lipids appear to form a uniform layer, whose formation may depend on the ability of lipids to disperse by flowing across the surface. Measurements of surface lipid viscosity are needed to test this hypothesis.

Low $-T_{\mathrm{m}}$ lipids might also affect chemical

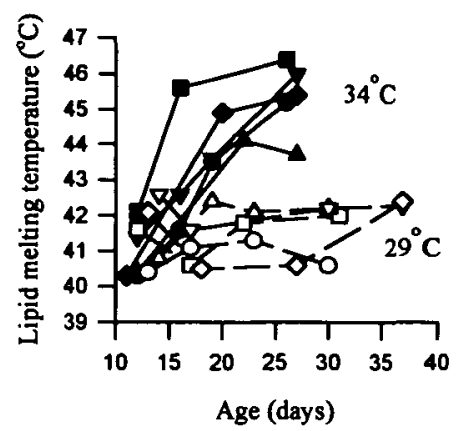

FIG. 7. Time course of thermal acclimation of cuticular lipids in the grasshopper, Melanoplus sanguinipes. Seven-day old nymphs were transferred from $27^{\circ} \mathrm{C}$ to $29^{\circ} \mathrm{C}$ or $34^{\circ} \mathrm{C}$ (open and filled symbols respectively), and shed skins were collected for determination of lipid melting temperatures. Each series of points indicates $T_{\mathrm{m}}$ values for successive instars from a single individual. From Gibbs and Mousseau (1994).

communication. Many insects use tactile pheromones in mate and colony recognition (Howard, 1993). The ability of a conspecific to detect a signal possibly could depend on whether the pheromone is embedded in a solid or fluid lipid matrix. The neurological basis for detection of volatile pheromones has been investigated in numerous insects, but mechanisms for detecting contact pheromones have been investigated in only a few cases (Städler et al., 1994).

Finally, perhaps the physical properties of cuticular lipids are not under active regulation. Acclimatory changes can be so slow that their utility in responding to environmental variation is questionable. For example, surface lipids of juvenile $M$. sanguinipes acclimate over a period of $2-3$ weeks, spanning 4 instars (Fig. 7). Other arthropods are certainly capable of changing their surface lipids much more rapidly than this. Surface lipids of houseflies consist primarily of methyl-branched alkanes (and very few alkenes) immediately after ecdysis, but alkenes are the dominant components a few days later. These sweeping changes include the production and deposition of lipid pheromones, a process that is under hormonal control (Blomquist et al., 1993). Changes in surface composition have also been observed in social insects (Howard, 1993). The causes and mecha- 
nisms whereby these latter changes occur are unknown, and may involve biosynthesis of new lipids or acquisition of them from other colony members.

Thus, cuticular lipids involved in chemical communication can be modified over a period of days, whereas the time course of thermal acclimation indicates a much slower restructuring. Perhaps temperature simply has different effects on the activities of biosynthetic enzymes, such that lipid composition and properties change as a result of altered channeling of precursors into different lipid classes. As long as the cuticle remains sufficiently impermeable, details of surface lipid composition and physical properties may not affect organismal water balance significantly. In this scenario, slow changes in surface lipid composition may simply reflect accumulated, non-adaptive changes due to the effects of temperature on biosynthetic enzymes. This non-adaptive model is consistent with data for $M$. sanguinipes and the desert fruit fly, D. mojavensis. Areas in which more work clearly is include the rates of turnover of cuticular lipids, and the effects of temperature on specific steps of lipid biosynthesis.

\section{Future questions in cuticular lipids}

Cuticular lipids are essential to the survival of terrestrial arthropods. However, most studies of the water-proofing abilities of these lipids have been correlative in nature, relying on comparative approaches or acclimatory variation. Ideally, one would like to manipulate surface lipid composition directly and examine the effects on water balance. As is rapidly becoming the case for membranes, the use of molecular techniques, in carefully chosen species, will become more common.

Many mutants for surface lipid biosynthesis have been discovered in plants (PostBeittenmiller, 1996), but genetic analysis of lipid synthesis and regulation in insects has only recently begun. This work has mainly focused on cuticular pheromones in Drosophila (Coyne et al., 1994; Scott, 1994; Ferveur et al., 1997), but physiologists can also use genetic approaches to study the consequences of lipid variation for water balance.

\section{CONCLUDING COMMENTS}

Lipids are defined by one of their physical properties (hydrophobicity). This property makes lipids an excellent barrier to the movement of water and other molecules into and out of cells and the whole organism, but other lipid properties can be functionally important. Despite considerable effort, it is not always apparent which lipid properties are crucial to physiological function. For example, intensive research into cell membranes has left us with several biophysical models from which to choose. A fundamental experimental problem is the complexity of lipid mixtures, and the fact that they are biosynthetically related. Thus, environmental factors that affect the production of one lipid class will affect the levels of other lipids.

Additional complexity arises from the multiple functions exhibited by many lipids. For example, pheromone components may make up the majority of cuticular lipids (Scott, 1994). In the case of cell membranes, unsaturated fatty acids are central to homeoviscous adaptation, are required to maintain the fluidity of depot fats in hibernating mammals (Frank, 1991, 1994), and are precursors for eicosanoids and hormones (Stanley and Howard, 1997). Thus, environmental effects or natural selection on one aspect of lipid biology will likely affect many other processes.

In spite of these limitations, the physical properties of lipids are clearly important in their biological functions. The biochemical processes responsible for changing membrane and cuticular lipid properties are largely known, but the regulation of these processes is very poorly understood. The techniques of molecular genetics have been used to identify loci involved in lipid biosynthesis, but these studies on their own are insufficient. Future advances will require the integration of biochemical, biophysical and molecular approaches, as well as investigations of the organismal effects of lipid variation.

\section{ACKNOWLEDGMENTS}

I thank Lisa Crockett for co-organizing the symposium, Jeannine Larabee for com- 
ments on the manuscript, Bryan Rourke for access to unpublished data, and the Society for Integrative and Comparative Biology for helping to fund the symposium. Funding for research and manuscript preparation was provided by NSF grant IBN-9317471, with travel support from the Ubu Endowment.

\section{REFERENCES}

Beament, J. W. L. 1945. The cuticular lipoids of insects. J. Exp. Biol. 21:115-131.

Behan-Martin, 1993. A near perfect temperature adaptation of bilayer order in vertebrate brain membranes. Biochim. Biophys. Acta 1151:216-222.

Bennett, A. F. 1997. Adaptation and the evolution of physiological characters. In W. H. Dantzler (ed.), Handbook of physiology: Section 13: Comparative physiology. Vol. 1, pp. 3-16. Oxford University Press, New York.

Blomquist, G. J., D. R. Nelson, and M. de Renobales 1987. Chemistry, biochemistry, and physiology of insect cuticular lipids. Arch. Insect Biochem. Physiol. 6:227-265.

Blomquist, G. J., J. A. Tillman-Wall, L. Guo, D. R Quilici, P. Gu, and C. Schal. 1993. Hydrocarbon and hydrocarbon derived sex pheromones in insects: Biochemistry and endocrine regulation. In D. W. Stanley-Samuelson and D. R. Nelson (eds.), Insect lipids: Chemistry, biochemistry and biology, pp. 317-351. University of Nebraska Press, Lincoln, Nebraska.

Buckner, J. S. 1993. Cuticular polar lipids of insects. In D. W. Stanley-Samuelson and D. R. Nelson (eds.), Insect lipids: Chemistry, biochemistry and biology, pp. 227-270. University of Nebraska Press, Lincoln, Nebraska.

Cantor, R. S. 1997. Lateral pressure in cell membranes: A mechanism for modulation of protein function. J. Phys. Chem. 101:1723-1725.

Carey, C. and J. R. Hazel. 1989. Diurnal variation in membrane lipid composition of Sonoran desert teleosts. J. Exp. Biol. 147:375-391.

Cevc, G. 1991. Isothermal lipid phase transitions. Chem. Phys. Lipids 57:293-307.

Chappell, M. A. 1983. Metabolism and thermoregulation in desert and montane grasshoppers. Oecologia 56:126-131.

Chong, P. L., P. A. G. Fortes, and D. M. Jameson 1985. Mechanisms of inhibition of $(\mathrm{Na}, \mathrm{K})$-ATPase by hydrostatic pressure studied with fluorescent probes. J. Biol. Chem. 260:14484-14490.

Cossins, A. R. 1994. Homeoviscous adaptation of biological membranes and its functional significance. In A. R. Cossins (ed.), Temperature adaptation of biological membranes, pp. 63-76. Portland Press, London.

Coyne, J. A., K. Mah, and A. P. Crittenden. 1994. Genetics of a pheromonal difference contributing to reproductive isolation in Drosophila. Science 265: 146I-1464.

Crockett, E. L. and J. R. Hazel. 1995. Cholesterol lev- els explain inverse compensation of membrane order in brush border but not homeoviscous adaptation in basolateral membranes from the intestinal epithelia of rainbow trout. J. Exp. Biol. 198:11051113.

Dey, I., T. Szegletes, C. Buda, J. Nemcsok, and T. Farkas. 1993. Fish erythrocytes as a tool to study temperature-induced responses in plasma membranes. Lipids 28:743-746.

Endler, J. A. 1986. Natural selection in the wild. Princeton University Press, Princeton, New Jersey.

Ferveur, J., M. Cobb, H. Boukella, and J. Jallon. 1996. World-wide variation in Drosophila melanogaster sex pheromone: Behavioural effects, genetic bases and potential evolutionary consequences. Genetica 97:73-80.

Ferveur, J., F. Savarit, C. J. O'Kane, Z. G. Sureau, R. J. Greenspan, and J. Jallon. 1997. Genetic feminization of pheromones and its behavioral consequences in Drosophila males. Science 276:15551558.

Frank, C. L. 1991. Adaptations for hibernation in the depot fats of a ground squirrel (Spermophilus beldingi). Can. J. Zool. 69:2707-2711.

Frank, C. L. 1994. Polyunsaturate content and diet selection by ground sqirrels (Spermophilus lateralis). Ecology 75:458-463.

Gibbs, A. 1995. Temperature, pressure and the sodium pump: The role of homeoviscous adaptation. $\ln \mathrm{P}$. W. Hochachka and T. P. Mommsen (eds.), Biochemistry and molecular biology of fishes, Vol. 5; pp. $197-212$.

Gibbs, A. G. 1997. Biochemistry at depth. In W. S. Hoar, D. J. Randall, and A. F. Farrell (eds.), Fish physiology, Vol. XVI, pp. 239-277.

Gibbs, A. and J. H. Crowe. 1991. Intra-individual variation in cuticular lipids studied using Fourier transform infrared spectroscopy. J. Insect Physiol. 37:743-748.

Gibbs, A. and T. A. Mousseau. 1994. Thermal acclimation and genetic variation in cuticular lipids of the lesser migratory grasshopper (Melanoplus sanguinipes): Effects of lipid composition on biophysical properties. Physiol. Zool. 67:1523-1543.

Gibbs, A. and J. G. Pomonis. 1995. Physical properties of insect cuticular hydrocarbons: The effects of chain length, methyl-branching and unsaturation. Comp. Biochem. Physiol. B 112:243-249.

Gibbs, A. and G. N. Somero. 1989. Pressure adaptation of $\mathrm{Na}^{+} / \mathrm{K}^{+}$-ATPase in gills of marine teleosts. $\mathrm{J}$. Exp. Biol. 143:475-492.

Gibbs, A. and G. N. Somero. 1990. Pressure adaptation of teleost gill $\mathrm{Na}^{+} / \mathrm{K}^{+}$-adenosine triphosphatase: Role of the lipid and protein moieties. J. Comp. Physiol. B 160:431-439.

Gibbs, A., T. A. Mousseau, and J. H. Crowe 1991. Genetic and acclimatory variation in biophysical properties of insect cuticle lipids. Proc. Natl. Acad. Sci. U.S.A. 88:7257-7260.

Gibbs, A. G., A. K. Chippindale, and M. R. Rose. 1997. Physiological mechanisms of the evolution of desiccation resistance in Drosophila melanogaster. J. Exp. Biol. 200:1821-1832. 
Gibbs, A. G., A. K. Louie, and J. A. Ayala. 1998. Effects of temperature on cuticular lipids and water balance in a desert Drosophila: Is thermal acclimation beneficial? J. Exp. Biol. 201:71-80.

Hadley, N. F. 1978. Cuticular permeability of desert tenebrionid beetles: Correlations with epicuticular hydrocarbon composition. Insect Biochem. 8:1722.

Hadley, N. F. 1985. The adaptive role of lipids in biological systems. Wiley-Interscience, New York. $319 \mathrm{pp}$.

Hadley, N. F. 1994. Water relations of terrestrial arthropods. Academic Press, San Diego, California.

Hadley, N. F. and T. D. Schultz. 1987. Water loss in three species of tiger beetles (Cicindela): Correlations with epicuticular hydrocarbons. J. Insect Physiol. 33:677-682.

Harris, W. E. 1985. Modulation of $\left(\mathrm{Na}^{+}, \mathrm{K}^{+}\right)$-ATPase activity by the lipid bilayer examined with dansylated phosphatidylserine. Biochemistry 24 : 2873-2883.

Hazel, J. R. 1995. Thermal adaptation in biological membranes: Is homeoviscous adaptation the explanation? Ann, Rev. Physiol. 57:19-42.

Hazel, J. R. and E. E. Williams. 1990. The role of alterations in membrane lipid composition in enabling physiological adaptation of organisms to their physical environment. Prog. Lipid Res. 29: 167-227.

Holdgate, M. W. and M. Seal. 1956. The epicuticular wax layers of the pupa of Tenebrio molitor $\mathrm{L}$. J. Exp. Biol. 33:82-106.

Howard, R. W. 1993. Cuticular hydrocarbons and chemical communication. In D. W. Stanley-Samuelson and D. R. Nelson (eds.), Insect lipids: Chemistry, biochemistry and biology, pp. 179226. University of Nebraska Press, Lincoln, Nebraska.

Kaneshiro, S. M. and D. S. Clark. 1995. Pressure effects on the composition and thermal behavior of lipids from the deep-sea thermophile Methanococcus jannaschii. J. Bacteriol. 177:3668-3672.

Lee, A. G. 1991. Lipids and their effects on membrane proteins: Evidence against a role for fluidity. Prog. Lipid Res. 30:323-348

Lee, J. A. C. and A. R. Cossins. 1990. Temperature adaptation of biological membranes: Differential homoeoviscous responses in brush-border and basolateral membranes of carp intestinal mucosa. Biochim. Biophys. Acta 1026:195-203

Loveridge, J. P. 1968. The control of water loss in Locusta migratoria migratorioides $\mathrm{R}$. \& F. I. Cuticular water loss. J. Exp. Biol. 49:1-13.

Macartney, A., B. Maresca, and A. R. Cossins. 1994. In: A. R. Cossins (ed.), Temperature adaptation of biological membranes, pp. 129-139. Portland Press, London.

Markow, T. A. and E. C. Toolson. 1990. Temperature effects on epicuticular hydrocarbons and sexual isolation in Drosophila mojavensis. In J. S. F. Barker, W. T. Starmer, and R. J. Maclntyre (eds.) Ecological and evolutionary genetics of Drosophila, pp. 315-331. Plenum Press, New York.

Poly, W. J. 1997. Nongenetic variation, genetic-envi- ronmental intcractions and altered gene expres sion. I. Temperature, photoperiod, diet, $\mathrm{pH}$ and sex-related effects. Comp. Biochem. Physiol. A 117:11-66.

Post, R. L. and I. Klodos. 1996. Interpretation of ex traordinary kinetics of $\mathrm{Na}^{+}-\mathrm{K}^{+}$-ATPase by a phase change. Am. J. Physiol. 271:C1415-C1423.

Post-Beittenmiller, D. 1996. Biochemistry and molecular biology of wax production in plants. Ann. Rev. Plant Physiol. Biochem. 47:405-430.

Raynard, R. S. and A. R. Cossins. 1991. Hoemoviscous adaptation and thermal compensation of sodium pump of trout erythrocytes. Am. J. Physiol. 260:R916-R924.

de Renobales, M., D. R. Nelson, and G. J. Blomquist 1991. Cuticular lipids. In K. Binnington and A. Retnakaran (eds.), Physiology of the insect epidermis, pp. 242-253. CSIRO Publications, East Melbourne Australia.

Robinson, N. C. and R. A. Capaldi. 1977. Interaction of detergents with cytochrome $c$ oxidase. Biochemistry 16:375-381

Scarlata, S. F. 1991. Compression of lipid membrane as observed at varying membrane positions. Biophys. J. 60:334-340.

Scott, D. 1994. Genetic variation for female mate discrimination in Drosophila melanogaster. Evolution 48:112-121.

Sinensky, M. 1974. Homeoviscous adaptation-a homeostatic process that regulates the viscosity of membrane lipids in Escherichia coli. Proc. Natl. Acad. Sci. U.S.A. 71:522-525.

Squier, T. C., D. J. Bigelow, and D. D. Thomas. 1988 Lipid fluidity directly modulates the overall protein rotational mobility of the Ca-ATPase in sarcoplasmic reticulum. J. Biol. Chem. 263:91789186.

Städler, E., B. Ernst, J. Hurter, and E. Boller. 1994 Tarsal contact chemoreceptor for the host marking pheromone of the cherry fruit fly, Rhagoletis cerasi: Responses to natural and synthetic compounds. Physiol. Entomol. 19:139-151

Stanley, D. W. and R. W. Howard. 1998. The biology of prostaglandins and related eicosanoids in invertebrates: Cellular, organismal and ecological actions. Amer. Zool. 38:369-381.

Stanley-Samuelson, D. W. and D. R. Nelson. 1993. Insect lipids: Chemistry, biochemistry and physiology. University of Nebraska Press, Lincoln, Nebraska.

Thompson, G. A. and Y. Nozawa. 1977. Tetrahymena: A system for studying dynamic membrane alterations within the eukaryotic cell. Biochim. Biophys. Acta 472:55-92.

Tiku, P. E., A. Y. Gracey, A. I. Macartney, R. J. Beynon, and A. R. Cossins. 1996. Cold-induced expression of $\Delta^{9}$-desaturase in carp by transcriptional and posttranslational mechanisms. Science 271: 815-818.

Toolson, E. C. 1982. Effects of rearing temperature on cuticle permeability and epicuticular lipid composition in Drosophila pseudoobscura. J. Exp. Zool. 222:249-253.

Tooison, E. C. and N. F. Hadley. 1979. Seasonal effects 
on cuticular permeability and epicuticular lipid composition in Centruroides sculpturatus Ewing 1928 (Scorpiones: Buthidae). J. Comp. Physiol. 129:319-325.

Toolson, E. C., T. R. White, and W. S. Glaunsinger. 1979. Electron paramagnetic resonance spectroscopy of spin-labelled cuticle of Centruroides sculpturatus (Scorpiones: Buthidae): Correlation with thermal effects on cuticular permeability. J. Insect Physiol. 25:271-275.

Vigh L., D. A. Los, I. Horvath, and N. Murata. 1993. The primary signal in the biological perception of temperature: Pd-catalyzed hydrogenation of membrane lipids stimulated the expression of the desA gene in Synechocystis PCC6803. Proc. Natl. Acad. Sci. U.S.A. 90:9090-9094.

Warren, G. B., P. A. Toon, N. J. M. Birdsall, A. G. Lee, and J. C. Metcalfe. 1974. Reversible lipid titrations of the activity of pure adenosine triphosphatase-lipid complexes. Biochemistry 13: 5501-5507.
Wigglesworth, V. B. 1945. Transpiration through the cuticle of insects. J. Exp. Biol. 21:97-114.

Williams, E. E. 1998. Membrane lipids: What membrane physical properties are conserved during physicochemically-induced membrane restructuring? Amer. Zool. 38:280-290.

Williams, E. E. and J. R. Hazel. 1994. Restructuring of plasma membrane phospholipids in isolated hepatocytes of rainbow trout during brief in vitro cold exposure. J. Comp. Physiol. B 164:600-608

Wodtke, E. 1981. Temperature adaptation of biological membranes: Compensation of the molar activity of cytochrome $c$ oxidase in the mitochondrial energy-transducing membrane during thermal acclimation of the carp (Cyprinus carpio L.) Biochim. Biophys. Acta 640:710-720.

Zakim, D., J. Kavecansky and S. Scarlata. 1992. Are membrane enzymes regulated by the viscosity of the membrane environment? Biochemistry. 31: 11589-11594.

Corresponding Editor: Gary C. Packard 